\section{IN LIGHT OF THE GOSPORT REVIEW, HOW TRANSPARENT IS OPIOID PRESCRIBING WITHIN HOSPICES?}

DA Cawley, I Kilonzo, D Di Francesco, K Skowronski. St Michael's Hospice

\subsection{6/spcare-2020-PCC.138}

Background With recent attention looking at opioid prescribing, the potential dangers with use in non-malignant pain conditions, increasing scrutiny on their indication, use and monitoring is now required. As supportive and palliative care verve into the earlier trajectories with conditions other than cancer, the traditional approach to escalating doses of strong opioids to manage mainly pain and breathlessness is being challenged emerging research.

Aim To explore the use of strong opioids within a hospice setting and to scrutinise the supporting documentation.

Methods Retrospective case-note review of patients within an inpatient and community hospice setting over a 3 month period.

Results 64 inpatient and 67 community case-notes were reviewed; $77 \%$ and $46 \%$ of patients having strong opioids when first reviewed by the hospice teams respectively. The most common opioids were morphine sulphate (26\% vs. $16 \%$ ), diamorphine $(20 \%$ vs. $4 \%)$ and oxycodone $(20 \%$ vs. $4 \%)$ with most inpatients (91\%) having had clear documentation to support the indication, use, dose changes and opioid rotation rationale. The proportion of patients that subsequently died $(51 / 64)$ within the inpatient unit, nearly all $(50 / 51=98 \%)$ had some form of subcutaneous opioid prescribed regularly at their time of death. The comparison within the community was possible given the multiple health providers involved in prescribing of end of life medications.

Conclusions The findings from this piece of work provide reassurance that strong opioids when used within an inpatient hospice setting are used proportionally, with clear indication and rationale for their use. The community based practice supported by hospices identities key concerns in terms of clarity and consistency in the prescribing of strong opioids in the last days of life. Given the multiple provider partners within the ever merging health landscape, clear and shared protocols identifying the responsible organisation to lead on opioid prescribing and their clear rationale will be key.

\section{CORNEA DONATION - OUR 2020 VISION}

Sarah Collins, Flora Langman, Simone Ali. Martlets Hospice, Brighton and Hove

10.1136/spcare-2020-PCC.139

Introduction There is a shortage of over 500 corneas for transplant each year in the UK. Most people up to age 85 are eligible to donate, even those with metastatic cancer. From March 2018, following a period of staff education, all eligible patients admitted to Martlets Hospice were asked routinely about cornea donation and offered an information leaflet to enable informed decision-making. Prior to this period, only one inpatient per year on average donated their corneas.

Method Evaluation of the project had two components:

1) Audit - the electronic records of all inpatient admissions from March to May 2018 were analysed to identify:

- eligibility for donation and the proportion of patients approached about this
- factors that may have hindered eligible and willing patients successfully donating corneas

2) Staff Opinion Survey - all staff working on the inpatient unit were emailed with an online questionnaire asking about their experiences and suggestions for improvement

Results Audit:

- 27 of the 56 eligible patients were asked

- 14 wanted to donate

- 6 went on to donate

Not informing next of kin and not adequately recording suitable alerts in the notes led to some missed donations

Staff opinion survey:

- 55 responses

- 34 respondents felt able to initiate conversations about cornea donation

Factors that would improve staff confidence were identified as: further training and more time for discussion with patients and families

\section{Conclusions}

1. Staff education in and engagement with cornea donation in the hospice setting can empower patients to make informed decisions

2. Sensitive conversations regarding the option of cornea donation are integral to advance care planning

3. Simple changes in practice and culture in our unit led to an increase in donated corneas in just 3 months.

4. Further improvements to the process are planned with a reaudit next year.

\section{NURSE-LED HOSPICE CLINIC. A CURATE'S EGG?}

Lynn Davies, Alice O'Connor. Severn Hospice

\subsection{6/spcare-2020-PCC. 140}

Severn Hospice comprises two separate units and notably different geographical areas, each with a team of communitybased 'outreach nurses' (linked to GP practices). Population totals 500,000 people, roughly equal per area. Telford \& Wrekin has a population density of $584 / \mathrm{km} 2$, Shropshire 136/ $\mathrm{km} 2$.

We aim to fulfil the national 'Ambitions' for palliative care and adopted a 'Living Well' strategy in 2017.

We offer nurse-led clinics within both hospices, an option given to all patients at initial triage. This initiative began in our Telford base and was evaluated positively by nursing staff. Shropshire clinics began in February 2019. We have evaluated these by reviewing everyone referred to the team from June September 2019 to establish if clinic appointments may have been suitable.

The results revealed a total of 186 patients accepted our nurse outreach service over the four months; 181 patients were visited in their own homes. Of these only 16 were identified by the assessing nurse as being able to attend a clinic appointment: the main themes suggesting inappropriateness: end of life care, too unwell to attend a clinic or transport, travel \& distance difficulties.

We also sent out clinic questionnaires in July 2019 to all twelve hospice outreach nurses, across both bases. Our questionnaires demonstrate significant differences by area: the more rural nurses noting difficulties with travel, including comments about cancelled and missed clinic appointments. 